\title{
Picolinic and Isonicotinic Acids: A Fourier Transform Microwave Spectroscopy Study
}

\author{
3 Isabel Peña, Marcelino Varela, Vanina G. Franco, Juan C. López, Carlos Cabezas, and José L. Alonso* \\ 4 Grupo de Espectroscopia Molecular (GEM), Edificio Quifima, Laboratorios de Espectroscopia y Bioespectroscopia, Unidad Asociada \\ 5 CSIC, Universidad de Valladolid, 47011 Valladolid, Spain
}

6

Supporting Information

\begin{abstract}
The rotational spectra of laser ablated picolinic and isonicotinic acids have been studied using broadband chirped pulse (CP-FTMW) and narrowband molecular beam (MB-FTMW) Fourier transform microwave spectroscopies. Two conformers of picolinic acid, s-cis-I and s-cis-II, and one conformer of isonicotinic acid have been identified through the analysis of their rotational spectra. The values of the inertial defect and the quadrupole coupling constants obtained for the most stable s-cis-I conformer of picolinic acid, evidence the formation of an $\mathrm{O}-\mathrm{H} \cdots \mathrm{N}$ hydrogen bond between the acid group and the endocyclic $\mathrm{N}$ atom. The stabilization provided by this hydrogen bond compensates the destabilization energy due to the adoption of a $-\mathrm{COOH}$ trans configuration in this conformer. Its $r_{\mathrm{s}}$ structure has been derived from the rotational spectra of several ${ }^{13} \mathrm{C},{ }^{15} \mathrm{~N}$, and ${ }^{18} \mathrm{O}$ species observed in their natural abundances. Mesomeric effects have been revealed by comparing the experimental values of the ${ }^{14} \mathrm{~N}$ nuclear quadrupole coupling constants in the isomeric series of picolinic, isonicotinic, and nicotinic acids.
\end{abstract}

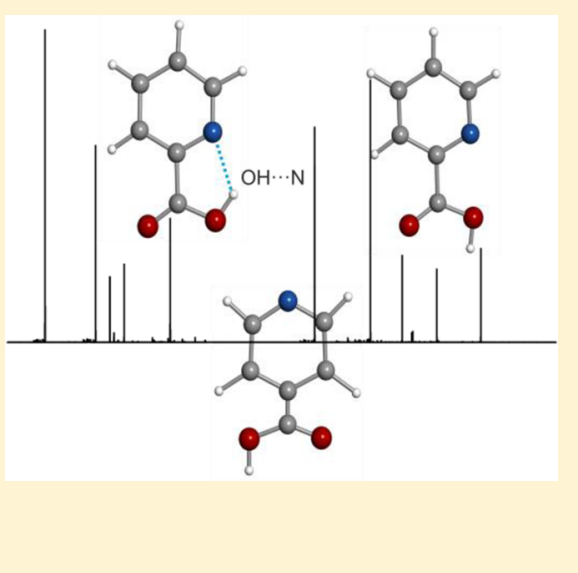

\section{$21 \square$ INTRODUCTION}

22 The two isomeric molecules picolinic (pyridine-2-carboxylic 23 acid) and isonicotinic (pyridine-4-carboxylic acid) acids $24\left(\mathrm{C}_{6} \mathrm{H}_{5} \mathrm{NO}_{2}\right)$ are pyridine derivatives, which have a great interest 25 due to their chemical and biological properties. The isonicotinic 26 acid is a main metabolite of isonicotinic acid hydrazide, which is 27 used as a therapeutic drug for tuberculosis. ${ }^{1}$ The picolinic acid 28 is an endogenous metabolite of L-tryptophan, which has been 29 detected in several biological mediums as blood serum, 30 cerebrospinal fluid, human milk, pancreatic juice, and intestinal 31 homogenates. $^{2-5}$ Picolinic acid is also known as a metal 32 chelating agent in the human body. They have been 33 investigated in condensed phases using IR, Raman, and X-ray 34 techniques, ${ }^{6-13}$ but none of them provide a detailed conforma35 tional picture about these isomeric species. Hence, the 36 experimental and theoretical vibrational spectra have been 37 interpreted in terms of the wavenumbers and intensities of 38 selected experimental bands, ${ }^{11}$ which have been compared and 39 discussed on the basis of the position of the nitrogen atom in 40 the aromatic ring. All results indicate that the structural 41 propensities observed in condensed phases are strongly biased 42 by interactions with the solvent or the crystal packing 43 forces. $^{12,13}$

44 The unambiguous characterization of the intrinsic conforma45 tional preferences can be achieved through the analysis of the 46 rotational spectra. Molecules placed in an isolated environment 47 such as that provided by the gas phase, free of the interactions 48 with the solvent, exhibit their intrinsic molecular properties. In 49 the present work we have investigated the rotational spectra of 50 the biological active molecules, picolinic and isonicotic acids, to obtain precise information on their conformation and structure. 51 The combination of narrowband molecular beam Fourier 52 transform microwave (MB-FTMW) spectroscopy and broad- 53 band chirped pulse Fourier transform (CP-FTMW) with laser 54 ablation (LA) vaporization provides a powerful experimental 55 tool, ${ }^{14,15}$ which allows the investigation of the rotational spectra 56 of solid biomolecules such as natural amino acids, ${ }^{16}{ }_{57}$ dipeptides, ${ }^{17}$ nucleic acid bases, ${ }^{18}$ and monosacharides ${ }^{19}$ in 58 the gas phase. We present here the first experimental rotational 59 studies of picolinic and isonicotinic acids using a combination 60 of the aforementioned techniques. The results have been 61 compared with those previously reported for nicotinic acid 62 (pyridine-3-carboxylic acid), in which two conformers, s-cis and 63 s-trans, have been recently unveiled. ${ }^{14}$

\section{EXPERIMENTAL SECTION}

65

Details of the Chirped-pulse Fourier transform microwave (CP- 66 FTMW) spectrometer coupled with a laser ablation source are 67 given elsewhere. ${ }^{14}$ Cylindrical rods of picolinic acid (m.p.: 13868 ${ }^{\circ} \mathrm{C}$ ) and isonicotinic acid (m.p.: $310^{\circ} \mathrm{C}$ ) samples were obtained 69 by pressing a mixture formed by fine powder of the substances 70 with a few drops of a commercial binder, in a hydraulic bench 71 press. They were vaporized in the ablation nozzle of the 72 spectrometer ${ }^{14}$ using the third harmonic $(355 \mathrm{~nm})$ of a ps 73 Nd:YAG laser (Ekspla, 20 ps $15 \mathrm{~mJ} /$ pulse). The vaporized 74 molecules seeded in the carrier gas $\mathrm{Ne}$ (stagnation pressure of 75

Received: September 29, 2014

Revised: November 4, 2014 
(a)

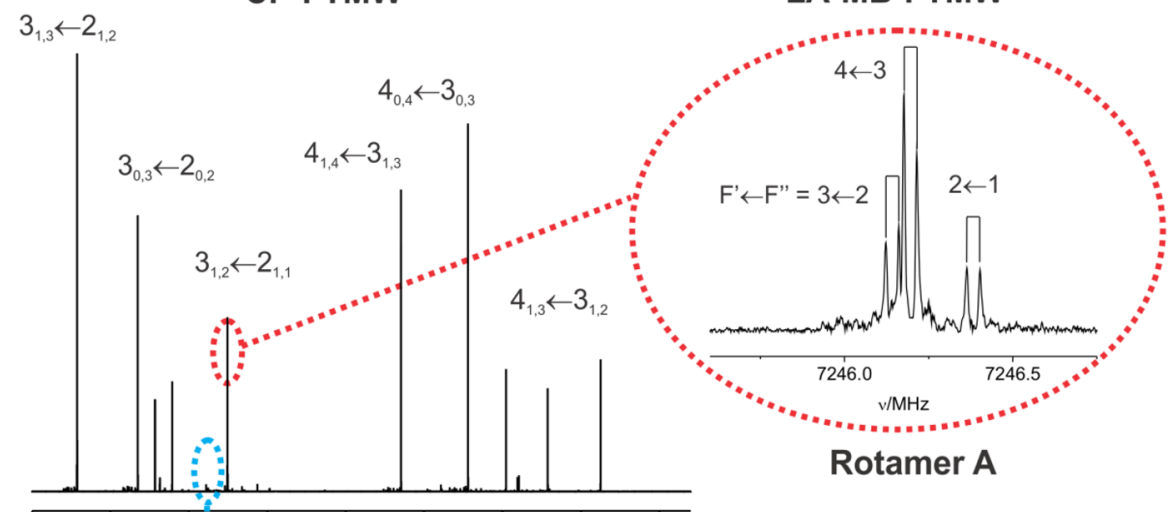

CP-FTMW

LA-MB-FTMW

(b)

6000

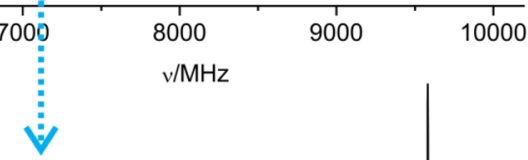

b)

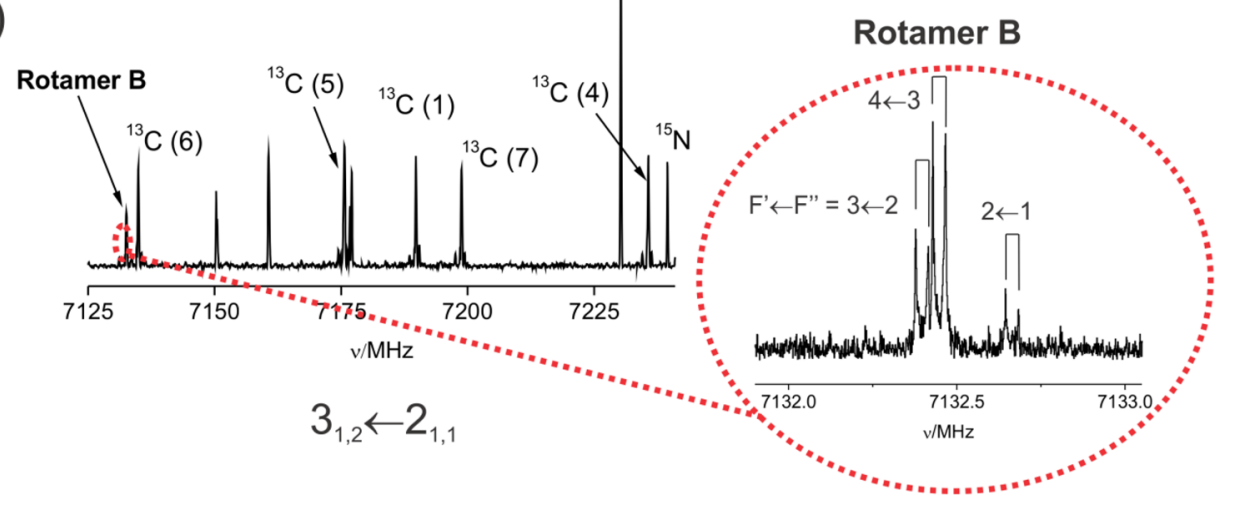

Figure 1. (a) CP-FTMW spectrum of laser ablated picolinic acid showing the groups of $\mu_{\mathrm{a}} \mathrm{R}$-branch transitions for rotamer A. The inlet shows the quadrupole hyperfine structure for the $3_{1,2} \leftarrow 2_{1,1}$ transition measured with the LA-MB-FTMW spectrometer. (b) The $3_{1,2} \leftarrow 2_{1,1}$ rotational transition for the different isotopic species of rotamer A observed in their natural abundances. The same rotational transition is observed for rotamer B. The resolved quadrupole hyperfine structure by LA-MB-FTMW spectroscopy is also shown in the inlet.

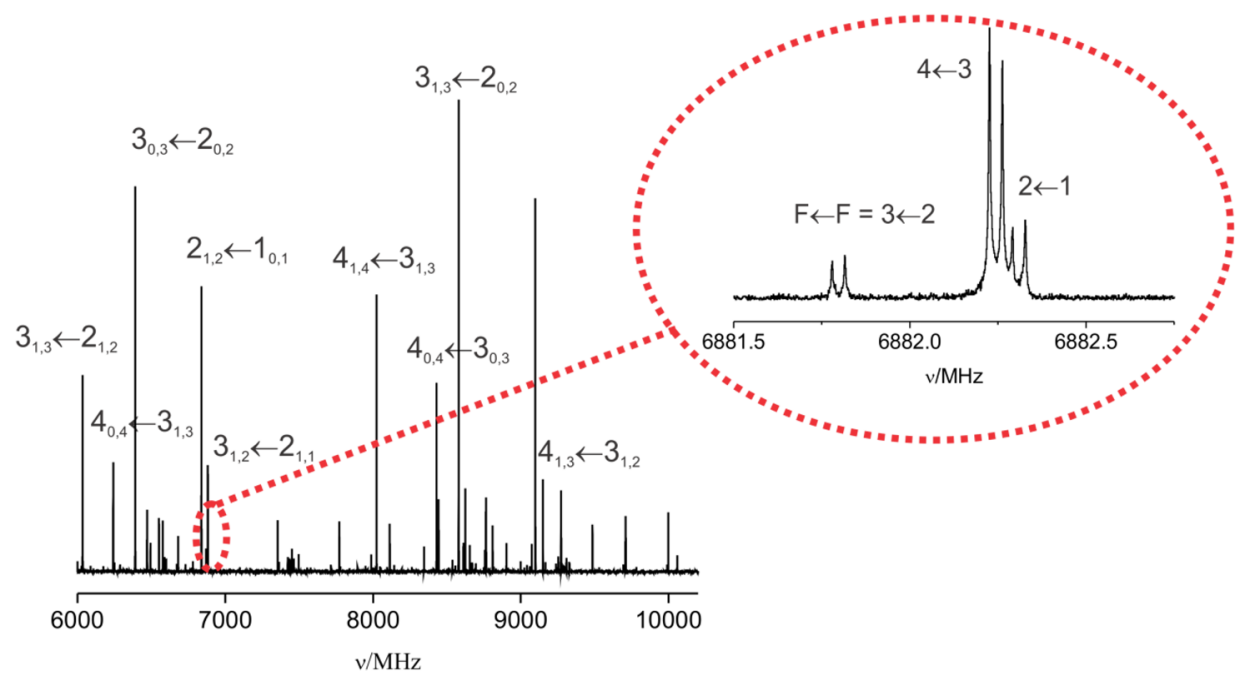

Figure 2. CP-FTMW spectrum of laser ablated isonicotinic acid and the quadrupole hyperfine structure for the $3_{12}-2_{11}$ rotational transition obtained by LA-MB-FTMW spectroscopy.

7610 bar) were expanded supersonically into the vacuum chamber 77 and probed by a microwave chirped pulse. Up to 60000 78 individual free induction decays (4 FIDs on each valve cycle) 79 for picolinic and isonicotinic acids were averaged in the time f1 domain rotational spectra from 6 to $10.2 \mathrm{GHz}$ shown in Figures $81 \mathrm{fl}$ 1 and 2. A Kaiser-Bessel window was applied to increase the $82 \mathrm{flf} 2$ baseline resolution.

Additional measurements were done using the higher 84 resolution of our LA-MB-FTMW spectrometer $^{15}$ to resolve 85 
86 the ${ }^{14} \mathrm{~N}$ nuclear quadrupole coupling hyperfine structure. This 87 spectrometer, which works in the $4-12 \mathrm{GHz}$ frequency region, 88 uses a laser ablation system with a ps-laser (Quantel, 35 ps 12 $89 \mathrm{~mJ} /$ pulse). The vaporized molecules were seeded in $\mathrm{Ne}$ at 15 90 bar of pressure and introduced into a Fabry-Pérot resonator to 91 form a supersonic jet. A short microwave radiation pulse $(0.3$ $92 \mu \mathrm{s})$ was then applied to macroscopically polarize the molecules 93 in the beam. The subsequent molecular emission signal was 94 collected and Fourier transformed to obtain the spectrum in the 95 frequency domain. In our setup, the microwave radiation travels 96 parallel to the axis of the resonator, and consequently, all 97 transitions appear as doublets because of the Doppler effect 98 (see inlets of Figure 1). The line width in this experiment is ca. $995 \mathrm{kHz}$, and the accuracy of the frequency measurements is 100 better than $3 \mathrm{kHz}$.

\section{RESULTS AND DISCUSSION}

102 Rotational Spectra Analysis. According to simple 103 considerations based on the expected coplanarity of the acid 104 and pyridine moieties, two possible arrangements s-cis or s-trans 105 of the $-\mathrm{COOH}$ group relative to the $\mathrm{N}$ atom can be anticipated 106 for picolinic acid. In the s-cis configuration, in which the $-\mathrm{OH}$ 107 group and the $\mathrm{N}$ atom are at the same side, two conformers s108 cis-I and s-cis-II have to be considered. Conformer s-cis-I 109 presents an $\mathrm{O}-\mathrm{H} \cdots \mathrm{N}$ intramolecular hydrogen bond with a 110 destabilizing $-\mathrm{COOH}$ trans configuration, while conformer s111 cis-II shows a $-\mathrm{COOH}$ cis configuration. Geometry optimiza112 tions for the three plausible conformers were carried out at the $113 \mathrm{MP} 2 / 6-311++\mathrm{G}(\mathrm{d}, \mathrm{p})^{20}$ level of theory with the Gaussian suite 114 of programs, $^{21}$ and the predicted rotational spectroscopic 115 constants are summarized in Table 1.

Table 1. Plausible Conformers of Picolinic Acid Together with the Calculated ${ }^{a}$ Spectroscopic Parameters and Energies

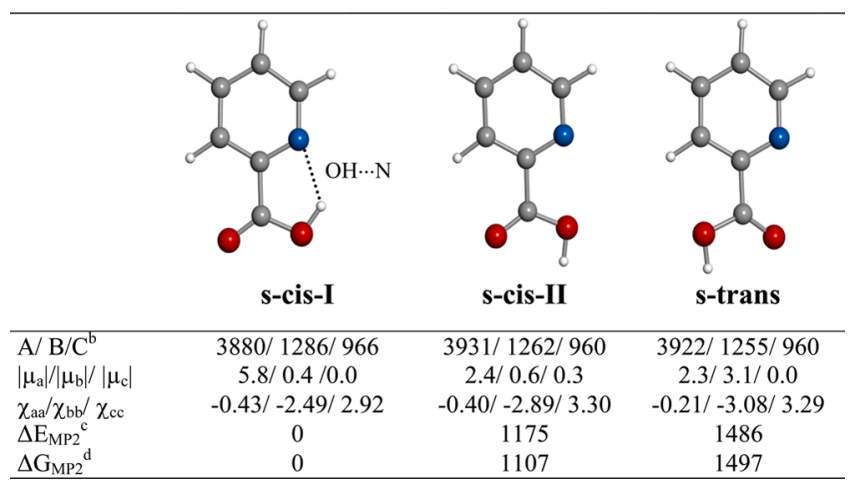

${ }^{a} \mathrm{Ab}$ initio calculations performed at the MP2/6-311++G(d,p) level of theory. ${ }^{b} \mathrm{~A}, \mathrm{~B}$, and $\mathrm{C}$ are the rotational constants (in $\mathrm{MHz}$ ); $\chi_{\mathrm{aa}} \chi_{\mathrm{bb}}$, and $\chi_{\mathrm{cc}}$ are the diagonal elements of the ${ }^{14} \mathrm{~N}$ nuclear quadrupole coupling tensor (in $\mathrm{MHz}$ ); $\left|\mu_{\mathrm{a}}\right|,\left|\mu_{\mathrm{b}}\right|$, and $\left|\mu_{\mathrm{c}}\right|$ are the electric dipole moment components (in D). ${ }^{c}$ Relative electronic energies calculated at $\mathrm{MP} 2 / 6-311++\mathrm{G}(\mathrm{d}, \mathrm{p})$ electronic energies (in $\left.\mathrm{cm}^{-1}\right) .{ }^{d} \mathrm{Gibbs}$ energies calculated at $298 \mathrm{~K}\left(\right.$ in $\left.\mathrm{cm}^{-1}\right)$.

116 The conformers of picolinic acid are near prolate asymmetric 117 tops with recognizable rotational spectrum patterns of $\mu_{\mathrm{a}}$-type 118 R-branch transitions separated by frequency intervals of 119 approximately $\mathrm{B}+\mathrm{C}$ values of rotational constants. Initial 120 inspection of the broadband CP-FTMW rotational spectrum 121 shown in Figure 1a soon revealed two sets of intense R-branch $122 \mu_{\mathrm{a}}$-type rotational transitions (with $J^{\prime} \leftarrow J^{\prime \prime}=3 \leftarrow 2$ and $4 \leftarrow 3$ ) 123 belonging to a first rotamer A. Rotational transitions exhibit the typical hyperfine structure arising from the interaction between 124 the electric quadrupole moment of the ${ }^{14} \mathrm{~N}$ nucleus $(\mathrm{I}=1)$ and 125 the electric field gradient created by all the molecular charges at 126 the site of this nucleus. Nevertheless, this hyperfine structure is 127 not well resolved by the CP-FTMW technique so only the 128 central frequencies were used in a rigid rotor analysis to obtain 129 a preliminary set of rotational constants employed in the initial 130 stage of the assignment procedure. $\mu_{\mathrm{b}}$ - and $\mu_{\mathrm{c}}$-type transitions 131 were also predicted but not observed. All the assigned 132 rotational transitions were subsequently submitted to the high 133 resolution of our LA-MB-FTMW spectrometer to resolve their 134 hyperfine structure (see inlet of Figure 1a). Hence, a total of 30135 hyperfine components belonging to nine $\mu_{\mathrm{a}}$-type R-branch 136 transitions were collected and fitted ${ }^{22}$ to a Hamiltonian $H=H_{\mathrm{R}} 137$ $+H_{\mathrm{Q}}$, where $H_{\mathrm{R}}$ is the rigid rotor Hamiltonian term and $H_{\mathrm{Q}}$ is 138 the nuclear quadrupole coupling interaction term. ${ }^{23}$ The 139 Hamiltonian was set up in the coupled basis set $I+J=F$, so 140 the energy levels involved in each transition are labeled with the 141 quantum numbers $J, K_{-1}, K_{+1}$, and $F$. The derived spectroscopic 142 parameters for rotamer A are listed in Table 2. Only the $143 \mathrm{t} 2$

Table 2. Spectroscopic Parameters Determined for Rotamers of A and B of Picolinic Acid

\begin{tabular}{lll} 
& rotamer A $(\mathrm{s}-c i s-\mathrm{I})$ & \multicolumn{1}{c}{ rotamer B (s-cis-II) } \\
$\mathrm{A}^{a}(\mathrm{MHz})$ & $3903.906(16)^{d}$ & $3958.969(17)$ \\
$\mathrm{B}(\mathrm{MHz})$ & $1290.82296(17)$ & $1268.13439(16)$ \\
$\mathrm{C}(\mathrm{MHz})$ & $970.41622(15)$ & $961.33930(15)$ \\
$\Delta_{\mathrm{c}}\left(\mu \AA^{2}\right)$ & $-0.18579(66)$ & $-0.47279(68)$ \\
$\chi_{\mathrm{aa}}(\mathrm{MHz})$ & $-0.5601(22)$ & $-0.5637(38)$ \\
$\chi_{\mathrm{bb}}(\mathrm{MHz})$ & $-2.5718(39)$ & $-3.030(16)$ \\
$\chi_{\mathrm{cc}}(\mathrm{MHz})$ & $3.1218(39)$ & $3.594(16)$ \\
$N^{b}$ & 30 & 24 \\
$\sigma^{c}(\mathrm{kHz})$ & 1.9 & 1.7
\end{tabular}

${ }^{a} \mathrm{~A}, \mathrm{~B}$, and $\mathrm{C}$ represent the rotational constants; $\Delta_{\mathrm{c}}=\left(\mathrm{I}_{\mathrm{c}}-\mathrm{I}_{\mathrm{a}}-\mathrm{I}_{\mathrm{b}}\right)=$ $-2 \Sigma_{\mathrm{i}} m_{\mathrm{i}} c_{\mathrm{i}}^{2}$ is the inertial defect; $\chi_{\mathrm{aa}}, \chi_{\mathrm{bb}}$, and $\chi_{\mathrm{cc}}$ are elements of the ${ }^{14} \mathrm{~N}$ nuclear quadrupole coupling tensor. ${ }^{b}$ Number of fitted hyperfine components. ${ }^{c}$ rms deviation of the fit. ${ }^{d}$ Standard error in parentheses in units of the last digit.

diagonal elements $\left(\chi_{\mathrm{aa}}, \chi_{\mathrm{bb}}\right.$, and $\left.\chi_{\mathrm{cc}}\right)$ of the quadrupole coupling 144 tensor $\chi$ were determined in the fit of the observed transitions 145 within the estimated accuracy of the frequency measurements. 146 The anisotropic quadrupole coupling tensor $\chi$ is related to the 147 electric field gradient tensor $q$ at the quadrupolar nucleus 148 through the nuclear quadrupole moment $e Q$ by $\chi=e Q q$. $\quad 149$

The high sensitivity reached in our experiment allowed us to 150 extend the spectral measurements to five monosubstituted ${ }^{13} \mathrm{C}, 151$ ${ }^{15} \mathrm{~N}$, and one ${ }^{18} \mathrm{O}$ species observed in their natural abundances 152 (approximately $1 \%, 0.4 \%$, and $0.2 \%$ ) for the A rotamer (see 153 Figure $1 \mathrm{~b}$ ). The analysis of the ${ }^{13} \mathrm{C}$ spectra was performed with 154 the same Hamiltonian used for the parent species. For the ${ }^{15} \mathrm{~N} 155$ species no quadrupole coupling interaction takes place so its 156 spectrum does not show hyperfine structure. The ${ }^{18} \mathrm{O}$ species 157 was only observed using the CP-FTMW spectrometer and thus 158 its quadrupole coupling hyperfine structure was not resolved. 159 The derived spectroscopic parameters for the isotopic species 160 are collected in Table 3.

Once the analysis of the parent and isotopic species of 162 rotamer A was completed and the lines discarded from the 163 rotational spectra, very weak $\mu_{\mathrm{a}}$-type R-branch transitions, 164 belonging to a second rotamer $\mathrm{B}$, were identified. The 165 intensities of the transitions are similar to those observed for 166 
Table 3. Experimental Spectroscopic Parameters for the Observed Isotopic Species of Rotamer A of Picolinic Acid

\begin{tabular}{|c|c|c|c|c|c|c|c|}
\hline & $\mathrm{C}_{1}$ & $\mathrm{C}_{4}$ & $\mathrm{C}_{5}$ & $\mathrm{C}_{6}$ & $\mathrm{C}_{7}$ & ${ }^{15} \mathrm{~N}$ & ${ }^{18} \mathrm{O}$ \\
\hline $\mathrm{A}^{a}(\mathrm{MHz})$ & $3860.328(16)$ & $3856.905(10)$ & $3863.912(12)$ & $3903.771(16)$ & 3903.719 (19) & $3865.356(17)$ & $3832.35(41)$ \\
\hline $\mathrm{B}(\mathrm{MHz})$ & $1281.01642(17)$ & $1289.77504(10)$ & $1278.27790(12)$ & $1269.79114(17)$ & 1281.84845 (19) & $1290.33767(18)$ & $1257.0929(64)$ \\
\hline $\mathrm{C}(\mathrm{MHz})$ & $962.17613(17)$ & $966.89405(10)$ & $960.85157(12)$ & $958.47311(16)$ & $965.32392(18)$ & $967.74067(18)$ & $946.9194(43)$ \\
\hline$\Delta_{c}\left(\mu \AA^{2}\right)$ & $-0.1843(7)$ & $-0.1843(4)$ & $-0.1840(5)$ & $-0.1858(7)$ & $-0.1858(8)$ & $-0.184(16)$ & $-0.185(19)$ \\
\hline$\chi_{\text {aa }}(\mathrm{MHz})$ & $-0.5366(27)$ & $-0.5744(25)$ & $-0.6259(29)$ & $-0.568(15)$ & $-0.5557(29)$ & & \\
\hline$\chi_{\mathrm{bb}}(\mathrm{MHz})$ & $-2.6033(65)$ & $-2.540(10)$ & $-2.516(12)$ & $-2.583(22)$ & $-2.5784(60)$ & & \\
\hline$\chi_{\mathrm{cc}}(\mathrm{MHz})$ & $3.1399(65)$ & $3.115(10)$ & $3.1418(12)$ & $3.151(22)$ & $3.1340(60)$ & & \\
\hline$N^{b}$ & 26 & 24 & 23 & 19 & 27 & 9 & 5 \\
\hline$\sigma^{c}(\mathrm{kHz})$ & 1.9 & 1.1 & 1.3 & 1.7 & 2.1 & 1.2 & 24.9 \\
\hline
\end{tabular}

${ }^{a} \mathrm{~A}, \mathrm{~B}$, and $\mathrm{C}$ represent the rotational constants; $\Delta_{\mathrm{c}}$ is the inertial defect; $\chi_{\mathrm{aa}}, \chi_{\mathrm{bb}}$, and $\chi_{\mathrm{cc}}$ are elements of the ${ }^{14} \mathrm{~N}$ nuclear quadrupole coupling tensor.

${ }^{b}$ Number of fitted hyperfine components. ${ }^{c}$ rms deviation of the fit. ${ }^{d}$ Standard error in parentheses in units of the last digit.

${ }_{167}^{13} \mathrm{C}$ species of rotamer $\mathrm{A}$, as can be seen in Figure $1 \mathrm{~b}$ for the $1683_{12}-2_{11}$ transition. No $\mu_{\mathrm{b}}$ - and $\mu_{\mathrm{c}}$-type transitions were 169 observed. A total of 24 hyperfine components corresponding 170 to the detected nine $\mu_{\mathrm{a}}$-type R-branch transitions were 171 measured using LA-MB-FTMW spectroscopy and analyzed 172 with the same Hamiltonian mentioned above. The final set of 173 rotational and nuclear quadrupole coupling constants are listed 174 in the second column of Table 2 .

175 The spectrum of isonicotinic acid has been investigated 176 following a similar procedure to that described for picolinic 177 acid. Differently from picolinic acid (ortho- $\mathrm{COOH}$ substituted), 178 in isonicotinic acid (para- $\mathrm{COOH}$ substituted), only the 179 conformer in Table 4 can be expected. Hence, the broadband

Table 4. Calculated ${ }^{a}$ and Experimental Spectroscopic Parameters for the Observed Rotamer of Isonicotinic Acid

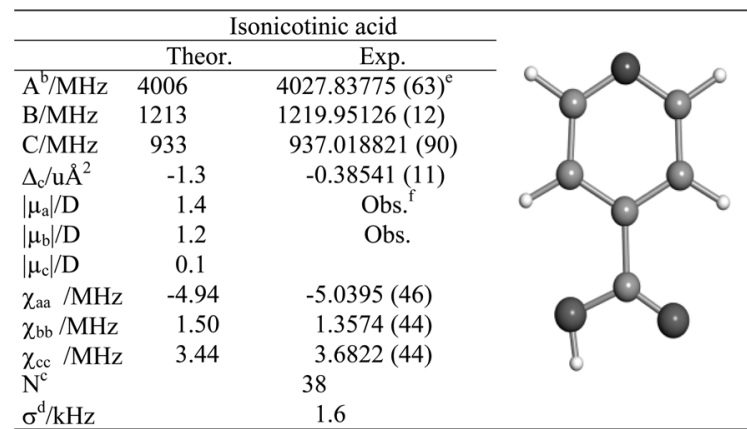

${ }^{a} \mathrm{Ab}$ initio calculations performed at the MP2/6-311++G(d,p) level of theory. ${ }^{b} \mathrm{~A}, \mathrm{~B}$, and $\mathrm{C}$ are the rotational constants; $\Delta_{\mathrm{c}}=\left(\mathrm{I}_{\mathrm{c}}-\mathrm{I}_{\mathrm{a}}-\mathrm{I}_{\mathrm{b}}\right)=$ $-2 \sum_{\mathrm{i}} m_{\mathrm{i}} c_{\mathrm{i}}^{2}$ is the inertial defect; $\left|\mu_{\mathrm{a}}\right|,\left|\mu_{\mathrm{b}}\right|$, and $\left|\mu_{\mathrm{c}}\right|$ are the electric dipole moment components; $\chi_{\mathrm{aa}}, \chi_{\mathrm{bb}}$, and $\chi_{\mathrm{cc}}$ are the diagonal elements of the ${ }^{14} \mathrm{~N}$ nuclear quadrupole coupling tensor. ${ }^{c}$ Number of fitted hyperfine components. ${ }^{d}$ rms deviation of the fit. ${ }^{e}$ Standard error in parentheses in units of the last digit. ${ }^{f}$ Observation of a- and b-type spectra.

180 CP-FTMW rotational spectrum of Figure 2 only shows sets of 181 intense R-branch $\mu_{\mathrm{a}^{-}}$and $\mu_{\mathrm{b}}$-type rotational transitions 182 attributable to one rotamer of isonicotinic acid. A total of 25 ${ }_{183}{ }^{14} \mathrm{~N}$ hyperfine components from nine $\mu_{\mathrm{a}}$-type R-branch 184 transitions and 13 corresponding to five $\mu_{\mathrm{b}}$-type R-branch 185 transitions were resolved and measured using LA-MB-FTMW 186 spectroscopy (see inlet of Figure 2). All hyperfine components 187 were fitted using the same Hamiltonian as described for 188 picolinic acid. The determined spectroscopic parameters are 189 also shown in Table 4. All frequency measurements for 190 picolinic and isonicotinic acids are provided as Supporting 191 Information (Table S1-S10).
Conformational Identification and Structure. Con- 192 formational identification is commonly achieved by comparing 193 the experimentally determined molecular properties with those 194 predicted ab initio. ${ }^{24}$ In particular, the rotational constants (A, 195 $\mathrm{B}, \mathrm{C})$, the ${ }^{14} \mathrm{~N}$ nuclear quadrupole coupling constants $\left(\chi_{\mathrm{aa}}, \chi_{\mathrm{bb}}, 196\right.$ $\left.\chi_{\mathrm{cc}}\right)$, and the values of the electric dipole moment components 197 along the principal inertial axis $\left(\mu_{\mathrm{a}}, \mu_{\mathrm{b}}, \mu_{\mathrm{c}}\right)$ should all be 198 consistent with ab initio values, even if only one of these tools is 199 acting as the discriminating element.

The values of the rotational constants depend on the mass 201 distribution around the principal inertial axes, so they are 202 commonly used as conclusive tool in the identification of the 203 observed species. In picolinic acid, the experimental values for 204 $\mathrm{A}$ and B rotamers in Table 2 are similar to those predicted for s- 205 cis-I, s-cis-II, and s-trans conformers in Table 1, thus making 206 their assignment difficult. The same works for the ${ }^{14} \mathrm{~N}$ nuclear 207 quadrupole coupling constants. This is reflected in nearly 208 identical quadrupole hyperfine patterns for both A and B 209 rotamers, as can be seen in the inlets of Figure 1 for the same 210 $3_{12}-2_{11}$ rotational transition. On this basis, the two observed 211 rotamers can be ascribed to the lowest energy conformers, but 212 an unambiguous identification to specific conformers cannot be 213 achieved using rotational and quadrupole coupling constants as 214 conformational tools.

The nonobservation of $\mu_{\mathrm{b}}$-type spectra for rotamers A and B 216 and the predicted low values of the $\mu_{\mathrm{b}}$ electric dipole moment 217 component for conformers s-cis-I and s-cis-II points to the 218 presence of these conformers in the supersonic expansion. On 219 the same basis, conformer s-trans should be discarded due to 220 the predicted high value of the $\mu_{\mathrm{b}}$ dipole moment component. 221 Conformer s-cis-I is predicted to be, by far, the most stable 222 conformer of picolinic acid in accordance with the intense 223 observed rotational spectrum for rotamer A. Additionally, the 224 predicted changes in the rotational constants going from s-cis-I 225 to s-cis-II are $\Delta \mathrm{A} \approx 50.9(55.1) \mathrm{MHz}, \Delta \mathrm{B} \approx-23.62(-22.7) 226$ $\mathrm{MHz}$, and $\Delta \mathrm{C} \approx-6.0(-9.1) \mathrm{MHz}$, which match nicely with 227 those experimentally observed (in parentheses) in going from 228 rotamer A to rotamer B. This allows the identification of 229 rotamers A and B as conformers s-cis-I and s-cis-II, respectively. 230 For isonicotinic acid, the predicted values of the rotational and 231 quadrupole coupling constants for the most stable species (see 232 Table 4) are in excellent agreement with those experimentally 233 observed.

234

The inertial defect values $\Delta_{c}$ in Table 2 obtained for the 235 observed conformers give a measure of the mass extension out 236 of the $a b$ plane. While for planar rigid molecules $\Delta_{c}$ should be 237 strictly zero, the ground state vibrational motions give rise to 238 inertial defect values, which are negative and close to zero. ${ }^{25-27} 239$ 
240 Hence, the planarity of all conformers is confirmed by the 241 experimental values of the inertial defect $\Delta_{c}$ (see Table 2$)$. The 242 differences in the experimental inertial defect values for 243 picolinic acid conformers s-cis-I ands-cis-II and isonicotinic 244 acid can be rationalized if we compare them with those 245 observed for related planar pyridine, ${ }^{28}$ benzoic acid, ${ }^{29}$ or 246 nicotinic acid. ${ }^{14}$ Hence, while in pyridine $\Delta_{c}$ is very small and 247 positive $\left(\Delta_{c}=0.039 \mu \AA^{2}\right)$, the negative values observed for the 248 rest of them collected in Table 6 indicates that out-of-plane 249 vibrations related to the presence of $-\mathrm{COOH}$ group have 250 dominant contributions.

251 A close look to the values in Table 6 shows that the inertial 252 defect for conformer s-cis-I $\left(\Delta_{c}=-0.186 \mu \AA^{2}\right)$ is roughly half 253 the value of benzoic $\left(\Delta_{\mathrm{c}}=-0.366 \mu \AA^{2}\right)$, nicotinic $\left(\Delta_{\mathrm{c}}=\right.$ $254-0.352 \mu \AA^{2}$ and $\Delta_{c}=-0.374 \mu \AA^{2}$ for conformers s-cis and s255 trans, respectively) and isonicotinic acids $\left(\Delta_{c}=-0.38541 \mu \AA^{2}\right)$, 256 which is, in turn, smaller than the inertial defect for conformer $257 \mathrm{~s}$-cis-II $\left(\Delta_{c}=-0.473 \mu \AA^{2}\right)$. In other words we can affirm that 258 the out of plane vibrational contributions of $-\mathrm{COOH}$ in s-cis-I 259 are smaller than in the rest of species. This is in accordance 260 with the observation of an intramolecular $\mathrm{O}-\mathrm{H} \cdots \mathrm{N}$ hydrogen 261 bond in the s-cis-I conformer, which limits in some extent the 262 amplitude of the out-of-plane $-\mathrm{COOH}$ vibration in this 263 conformer. The fact that the $\Delta_{c}$ value observed for conformer 264 s-cis-II is slightly higher than those of benzoic acid, nicotinic 265 acid, or isonicotinic acid may be explained in terms of the $\mathrm{N} \cdots \mathrm{O}$ 266 repulsions, which would contribute to increase the amplitude of 267 the out-of-plane $-\mathrm{COOH}$ vibrations in this conformer. These $268 \mathrm{~N} \cdots \mathrm{O}$ repulsions are not present in the meta and para positions 269 of the $-\mathrm{COOH}$ groups in nicotinic and isonicotinic acids.

270 Finally, it should be noted that the value of the inertial defect 271 is practically invariant in all the isotopic species (Table 3), 272 which constitutes an additional test for the planarity of the s-cis273 I conformer. This isotopic information was used to determine 274 the coordinates of the substituted atoms in the principal axis 275 system using Kraitchmann's substitution method ${ }^{30-32}$ and to 276 determine the bond distances and angles of the heavy atom 277 skeleton listed in Table 5.

278 Quadrupole Coupling Constants: Intramolecular 279 Hydrogen Bonding and Mesomeric Effect. An inspection 280 of the experimental values of the nuclear quadrupole coupling 281 constants could reveal interesting information on the different

Table 5. Substitution Coordinates and $r_{\mathrm{s}}$ Structure for Conformer s-cis-I of Picolinic Acid

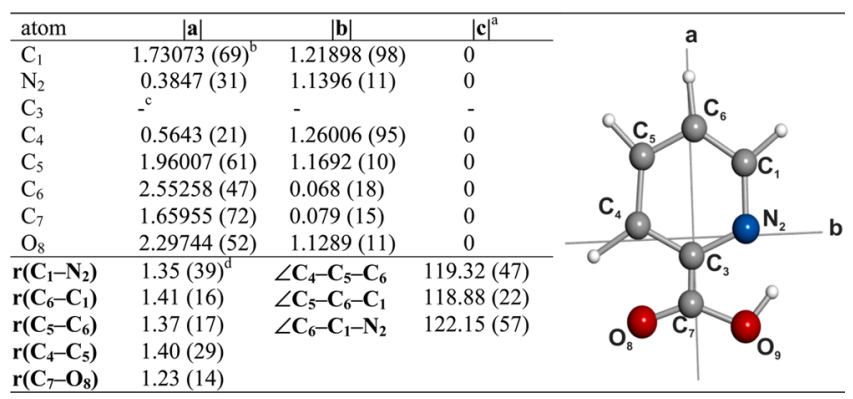

${ }^{a}$ Planar structure has been assumed. ${ }^{b}$ Principal inertial axis coordinates in $\AA$; derived errors in parentheses in units of the last digit; These were calculated according to Constains formula: $\sigma(x)=K /|x| ; \sigma(x)$ is the error in the $x$ coordinate and $K=0.0012 \AA^{2}$. ${ }^{c}$ The rotational transitions of the ${ }^{13} \mathrm{C}$ species of the $\mathrm{C}_{3}$ carbon atom are so close to those of the parent species that become undetectable. ${ }^{d}$ Distances in $\AA$ and angles in degrees. nature of the observed conformers. The ${ }^{14} \mathrm{~N}$ nucleus present in 282 all the conformers possess a nonzero quadrupole moment $(\mathrm{I}=283$ 1) owing to a nonspherical distribution of the nuclear charge, 284 which interacts with the electric field gradient created by the 285 rest of the molecule at the site of the nucleus. The associated 286 experimentally determinable molecular properties are the 287 diagonal elements $\left(\chi_{\mathrm{aa}}, \chi_{\mathrm{bb}}, \chi_{\mathrm{cc}}\right)$ of the nuclear quadrupole 288 coupling tensor that are directly related to the electronic 289 environment around the nitrogen nucleus referred to the 290 principal inertial axes. For planar molecules, like those of 291 isomeric picolinic, nicotinic, ${ }^{14}$ and isonicotinic acids, the $\chi_{\mathrm{cc}} 292$ element of the quadrupole coupling tensor is coincident with 293 one of the elements of the principal quadrupole coupling tensor 294 and provides information on the electric field gradient along the 295 direction of an axis perpendicular to the aromatic ring. For 296 pyrrolic nitrogens $(-\mathrm{N}<) \chi_{c c}$ is negative. ${ }^{33-35}$ In contrast, 297 pyridinic nitrogens $(-\mathrm{N}=)$ have positive values of $\chi_{\mathrm{cc}}{ }^{34-36} 298$ Conformerss-cis-I and s-cis-II of picolinic acid have positive 299 values for $\chi_{\mathrm{cc}}$ indicating their pyridinic nature. However, while 300 conformer s-cis-II presents a value for $\chi_{\mathrm{cc}}$ of $3.594 \mathrm{MHz}$ very 301 close to that of pyridine (see Table 6), a markedly lower value 302 t6 of $3.122 \mathrm{MHz}$ is found for the s-cis-I conformer. This difference 303 cannot be attributed to possible changes in the orientation of 304 the principal inertial axes; both conformers differ only in the 305 orientation of the hydroxyl group. It must be ascribed to the 306 formation of an intramolecular hydrogen bond $\mathrm{O}-\mathrm{H} \cdots \mathrm{N}$ in the 307 s-cis-I conformer, which increase slightly the pyrrolic character 308 of the nitrogen nucleus by diminishing the $\chi_{\mathrm{cc}}$ value. 309

A more detailed analysis of the nuclear quadrupole coupling 310 constants shows small discrepancies in the $\chi_{\mathrm{cc}}$ values in passing 311 from ortho- and para-COOH substituted species to the meta- 312 $\mathrm{COOH}$ ones. Hence, in the case of s-cis-II picolinic (ortho- 313 $\mathrm{COOH}$ substituted) and isonicotinic (para-COOH substi- 314 tuted) acids, one observes a small increase of the $\chi_{\mathrm{cc}}$ with 315 respect to the values of nicotinic s-cis or s-trans ${ }^{14}$ (meta-COOH 316 substituted), which adopt practically the same value as for 317 pyridine (see Table 6). Here an effect based on the exchange 318 interaction of the lone electron pairs of the $-\mathrm{COOH} 319$ substituent with the $\pi$ electrons of the pyridine ring ${ }^{36}$ can be 320 considered. This type of electron shift is termed mesomeric 321 effect $^{37,38}$ and is signified by $-\mathrm{M}$ or $+\mathrm{M}$, depending on whether 322 the electron shift takes place from the aromatic ring to the 323 substituent $(-\mathrm{M})$ or in the reverse direction $(+\mathrm{M})$. In the case 324 of the isomeric series of picolinic, nicotinic, and isonicotinic 325 acids, the electron distribution shift is in favor of the carboxylic 326 group ( $-\mathrm{M})$, extracting electrons from the $\pi$-ring system in 327 ortho and para positions (see Figure 3), which is reported by a $328 \mathrm{f} 3$ small but noticeable increase in the $\chi_{\mathrm{cc}}$ values of about 0.2329 $\mathrm{MHz}$. For the s-cis-I conformer, the decrease in the value of $\chi_{\mathrm{cc}} 330$ with respect to s-cis-II conformer might be due to a negative 331 polarization at the $\mathrm{N}$ atom arising from the formation of a 332 $-\mathrm{COOH} \cdots \mathrm{N}$ hydrogen bond.

\section{CONCLUSIONS}

334

In this work, two of the three plausible conformers of picolinic 335 acid and the only plausible conformer for isonicotinic acid have 336 been observed. For the most stable conformer of picolinic acid 337 s-cis-I the heavy atom skeleton structure has been determined 338 from the observation of ${ }^{13} \mathrm{C},{ }^{18} \mathrm{O}$, and ${ }^{15} \mathrm{~N}$ isotopologues in 339 natural abundance using the CP-FTMW spectrometer. The 340 formation of an intramolecular $\mathrm{O}-\mathrm{H} \cdots \mathrm{N}$ hydrogen bond in the 341 most stable conformer of picolinic acid has been also revealed 342 by its signatures in the quadrupole coupling constants and the 343 
Table 6. Experimental Inertial Defects $\left(\mathrm{in} \mu \AA^{2}\right.$ ) and ${ }^{14} \mathrm{~N}$ Quadrupole Coupling Constants (in MHz) of Picolinic and Isonicotinic Acids Compared to Those of Nicotinic Acid, Benzoic Acid, and Pyridine

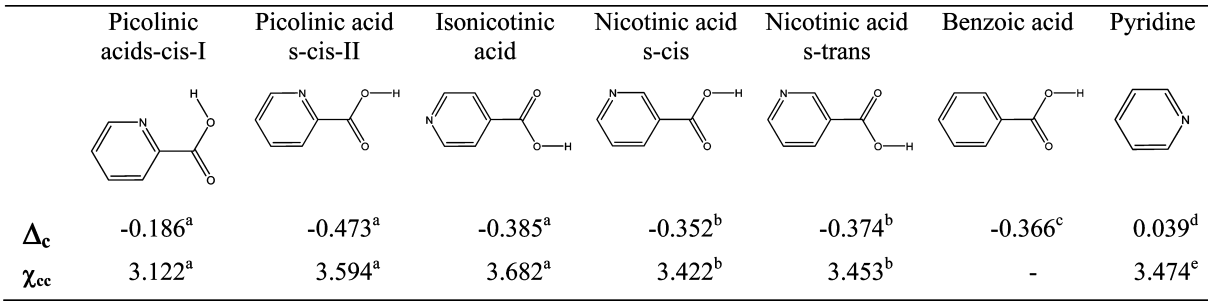

${ }^{a}$ Present work. ${ }^{b}$ From ref $14 .{ }^{c}$ From ref $29 .{ }^{d}$ From ref $28 .{ }^{e}$ From ref 36.<smiles>O=C(O)c1cc[n+]([O-])cc1</smiles><smiles>O=C(O)c1ccccc1</smiles>

Figure 3. Resonance withdrawing effect in picolinic (left) and isonicotinic (right) acids.

344 vibrational contributions to the inertial defect values. The 345 formation of this hydrogen bond forces the $-\mathrm{COOH}$ group to 346 adopt a trans configuration, considerably less stable than its 347 usual cis configuration. The stabilization energy provided by 348 this hydrogen bond interaction thus compensates the 349 destabilization due to the adoption of a $-\mathrm{COOH}$ trans 350 configuration.

351 The role of the quadrupole coupling constants has been also 352 found to be essential to detect the subtle effects of exchange 353 interactions in aromatic systems. Hence, mesomeric effect from $354-\mathrm{COOH}$ group in ortho and para positions has been unveiled 355 in picolinic and isonicotinic acids by comparing the $\chi_{\mathrm{cc}}$ values of 356 the quadrupole coupling constants to those found for nicotinic 357 acid and pyridine molecules.

358 It has been shown that the combination of laser ablation with 359 the chirped-pulse Fourier transform microwave spectroscopy 360 (CP-FTMW) provides an efficient tool in the analysis of the 361 rotational spectra of biomolecules with high melting points. 362 This technique is complementary to LA-MB-FTMW spectros363 copy with superior resolution to resolve the hyperfine structure. 364 Both techniques constitute sensitive tools for the structure 365 determination of biomolecules.

\section{ASSOCIATED CONTENT}

\section{S Supporting Information}

368 Complete ref 21, list of measured transitions for the observed 369 conformers of picolinic and isonicotinic acids, and ab initio 370 coordinates and structural parameters for conformer s-cis-I of 371 picolinic acid. This material is available free of charge via the 372 Internet at http://pubs.acs.org.

\section{$373 \square$ AUTHOR INFORMATION}

\section{Corresponding Author}

375 *E-mail: jlalonso@qf.uva.es. Tel: +34 983186345.

376 Notes

377 The authors declare no competing financial interest.

\section{ACKNOWLEDGMENTS}

378

This work has been supported by the Ministerio de Ciencia e 379 Innovación (Grants CTQ2010-19008 and Consolider-Ingenio 380 2010 CSD2009-00038) and Junta de Castilla y León (Grant 381 VA175U13). C.C. thanks the Junta de Castilla y León for a 382 postdoctoral contract (Grant CIP13/01).

(1) Iwata, K.; Ogata, S.; Okumura, K.; Taguchi, H. Induction of 385 Differentiation in Human Promyelocytic Leukemia HL-60 Cell Line 386 by Niacin-related Compounds. Biosci. Biotechnol. Biochem. 2003, 67387 (5), 1132-1135.

(2) Grant, R. S.; Coggan, S. E.; Smythe, G. A. The Physiological 389 Action of Picolinic Acid in the Human Brain. Int. J. Tryptophan Res. 390 2009, 2, 71-79.

(3) Dazzi, C.; Candiano, G.; Massazza, S.; Ponzetto, A.; Varesio, L. 392 New High-Performance Liquid Chromatographic Method for the 393 Detection of Picolinic Acid in Biological Fluids. J. Chromatogr. B: 394 Biomed. Sci. Appl. 2001, 751, 61-68.

(4) Smythe, G. A.; Braga, O.; Brew, B. J.; Grant, R. S.; Guillemin, G. 396 J.; Kerr, S. J.; Walker, D. W. Concurrent Quantification of Quinolinic, 397 Picolinic, and Nicotinic Acids Using Electron-Capture Negative-Ion 398 Gas Chromatography Mass Spectrometry. Anal. Biochem. 2002, 301, 399 $21-26$.

(5) Rebello, T.; Lonnerdal, B.; Hurley, L. S. Picolinic Acid in Milk, 40 Pancreatic Juice, and Intestine: Inadequate for Role in Zinc 402 Absorption. Am. J. Clin. Nutr. 1982, 35, 1-5.

(6) Paris, M.; Thomas, G.; Merlin, J. C. Structure de l'Acide 404 Picolique. Bull. Soc. Chim. France 1961, 707-719. 405

(7) Hamann, S. D. The Influence of Pressure on the Infrared Spectra 406 of Hydrogen-Bonded Solids. The Formation of Fermi Resonance 407 'Windows'. Aust. J. Chem. 1977, 30, 71-9.

(8) Imai, Y.; Kurokawa, Y.; Hara, M.; Fukushima, M. Observation of 409 SERS of Picolinic Acid and Nicotinic Acid Using Cellulose Acetate 410 Films Doped with Ag Fine Particles. Spectrochim. Acta, Part A 1997, 411 53, 1697-1700.

(9) Spinner, E. A Strongly Hydrogen-Bonded Molecular Solid, 413 Isonicotinic Acid: Raman Spectra of the - $\mathrm{C}^{\prime \prime} \mathrm{O}, \mathrm{H}$ and $-\mathrm{C}^{\prime \prime} \mathrm{O}, \mathrm{D}$ Species 414 and Infrared and Raman Spectra of the $-\mathrm{C}^{\prime \prime} \mathrm{O}, \mathrm{H}$ Acid. J. Phys. Chem. 415 1988, 92, 3379-3386.

(10) Park, S. M.; Kim, K.; Kim, M. S. Raman Spectroscopy of 417 Isonicotinic Acid Adsorbed onto Silver Sol Surface. J. Mol. Struct. 1994, 418 328, 169-178.

419

(11) Koczon, P.; Dobrowolski, J. Cz.; Lewandowski, W.; Mazurek, A. 420 P. Experimental and Theoretical IR and Raman Spectra of Picolinic, 421 Nicotinic and Isonicotinic Acids. J. Mol. Struct. 2003, 65, 89-95. 422

(12) Takusagawa, F.; Shimada, A. The Crystal Structure of Picolinic 423 Acid. Chem. Lett. 1973, 1089-1090. 424

(13) Takusagawa, F.; Shimada, A. Isonicotinic Acid. Acta Crystallogr. 425 1976, B32, 1925.

426

(14) Mata, S.; Peña, I.; Cabezas, C.; López, J. C.; Alonso, J. L. A 427 Broadband Fourier-Transform Microwave Spectrometer with Laser 428 Ablation Source: The Rotational Spectrum of Nicotinic Acid. J. Mol. 429 Spectrosc. 2012, 280, 91-96. 
431 (15) Alonso, J. L.; Pérez, C.; Sanz, M. E.; López, J. C.; Blanco, S. 432 Seven Conformers Of L-Threonine in the Gas Phase: A LA-MB433 FTMW Study. Phys. Chem. Chem. Phys. 2009, 11, 617-627.

434 (16) Cabezas, C.; Varela, M.; Peña, I.; Mata, S.; López, J. C.; Alonso, $435 \mathrm{~J}$. L. The Conformational Locking of Asparagine. Chem. Commun. 436 2012, 48, 5934-5936 and references therein..

437 (17) Puzzarini, C.; Biczysko, M.; Barone, V.; Largo, L.; Peña, I.; 438 Cabezas, C.; Alonso, J. L. Accurate Characterization of the Peptide 439 Linkage in the Gas Phase: A Joint Quantum-Chemical and Rotational 440 Spectroscopy Study of the Glycine Dipeptide Analogue. J. Phys. Chem. 441 Lett. 2014, 5, 534-540 and references therein..

442 (18) Alonso, J. L.; Peña, I.; López, J. C.; Vaquero, V. Rotational 443 Spectral Signatures of Four Tautomers of Guanine. Angew. Chem., Int. 444 Ed. 2009, 48, 6141-6143 and references therein..

445 (19) Alonso, J. L.; Lozoya, M.; Peña, I.; López, J. C.; Cabezas, C.; 446 Mata, S.; Blanco, S. The Conformational Behaviour of Free D-Glucose447 at Last. Chem. Sci. 2014, 5, 515-522.

448 (20) Møller, C.; Plesset, M. S. Note on an Approximation Treatment 449 for Many-Electron Systems. Phys. Rev. 1934, 46, 618-622.

450 (21) Frisch, M. J.; Trucks, G. W.; Schlegel, H. B.; Scuseria, G. E.; 451 Robb, M. A.; Cheeseman, J. R.; Scalmani, G.; Barone, V.; Mennucci, 452 B.; Petersson, G. A.; et al. Gaussian 09, revision B.01; Gaussian, Inc.: 453 Wallingford, CT, 2010.

454 (22) Pickett, H. M. The Fitting and Prediction of Vibration-Rotation 455 Spectra with Spin Interactions. J. Mol. Spectrosc. 1991, 148, 371.

456 (23) Gordy, W.; Cook, R. L. In Microwave Molecular Spectra, 3rd ed.; 457 Weissberg, A., Ed.; Techniques of Chemistry; John Wiley \& Sons Inc.: 458 New York, 1984; Vol. XVIII.

459 (24) Peña, I.; Sanz, M. E.; López, J. C.; Alonso, J. L. Preferred 460 Conformers of Proteinogenic Glutamic Acid. J. Am. Chem. Soc. 2012, 461 134, 2305-2312 and references therein..

462 (25) Oka, T. On Negative Inertial Defect. J. Mol. Struct. 1995, 352/ $463353,225-233$.

464 (26) Jagod, M.-F.; Oka, T. Inertial Defects of Planar Symmetric Top 465 Molecules. J. Mol. Spectrosc. 1990, 139, 313-327.

466 (27) Oka, T.; Morino, Y. Inertial Defect. Part III. Inertia Defect and 467 Planarity of Four-Atomic Molecules. J. Mol. Spectrosc. 1963, 11, 349468367.

469 (28) Ye, E.; Bettens, R. P. A.; De Lucia, F. C.; Petkie, D. T.; Albert, S. 470 Millimeter and Submillimeter Wave Rotational Spectrum of Pyridine 471 in the Ground and Excited Vibrational States. J. Mol. Spectrosc. 2005, 472 232, 61-65.

473 (29) Onda, M.; Asai, M.; Takise, K.; Kuwae, K.; Hayami, K.; Kuroe, 474 A.; Mori, M.; Miyazaki, H.; Suzuki, N.; Yamaguchi, I. Microwave 475 Spectrum of Benzoic Acid. J. Mol. Struct. 1999, 482-483, 301-303.

476 (30) Kraitchmann, J. Determination of Molecular Structure from 477 Microwave Spectroscopic Data. Am. J. Phys. 1953, 21, 17-24.

478 (31) Costain, C. C. Determination of Molecular Structures from 479 Ground State Rotational Constants. J. Chem. Phys. 1958, 29, 864.

480 (32) Van Eijck, B. P. Influence of Molecular Vibrations on 481 Substitution Coordinates. J. Mol. Spectrosc. 1982, 91, 348-362.

482 (33) Bohn, R. K.; Hillig, K. W., II; Kuczkowski, R. L. Pyrrole-Argon: 483 Microwave Spectrum, Structure, Dipole Moment, and ${ }^{14} \mathrm{~N}$ Quadrupole 484 Coupling Constants. J. Phys. Chem. 1989, 93, 3456-3459.

485 (34) Tanjaroon, C.; Subramanian, R.; Karunatilaka, C.; Kukolich, S. 486 G. Microwave Measurements of ${ }^{14} \mathrm{~N}$ and D Quadrupole Coupling for 487 (Z)-2-Hydroxypyridine and 2-Pyridone Tautomers. J. Phys. Chem. A 488 2004, 108, 9531-9539.

489 (35) Stolze, M.; Sutter, D. H. The Rotational Zeeman Effect of 490 Pyrazole and Imidazole. Z. Naturforsch. A 1987, 42a, 49-56.

491 (36) Heineking, N.; Dreizler, H.; Schwarz, R. Nitrogen and 492 Deuterium Hyperfine Structure in the Rotational Spectra of Pyridine 493 and [4-D] Pyridine. Z. Naturforsch. A 1986, 41a, 1210-1213.

494 (37) Schmidt, A. Biologically Active Mesomeric Betaines and 495 Alkaloids Derived from 3-Hydroxypyridine, Pyridin- $\mathrm{N}$-oxide, Nicotinic 496 Acid and Picolinic Acid. Three Types of Conjugation and their 497 Consequences. Curr. Org. Chem. 2004, 8, 653.

498 (38) McMurry, J. E. Organic Chemistry, 8th ed; Cengage Learning: 499 Independence, KY, 2012. 